\title{
Acute Bacterial Prostatitis in Humans: Current Microbiological Spectrum, Sensitivity to Antibiotics and Clinical Findings
}

\author{
Vincent Nagy Dušan Kubej \\ Department of Urology, P.J. Šafárik University, Medical Faculty and L. Pasteur University Hospital, SNP 1, \\ Košice, Slovak Republic
}

\section{Key Words}

Acute bacterial prostatitis - Microbial spectrum - Sensitivity to antibiotics

\begin{abstract}
Objective: To assess the current microbiological profile and sensitivity to antibiotics in patients with acute bacterial prostatitis (ABP). Patients and Methods: In the period 20032010, we treated 192 ABP patients, aged from 18 to 85 years (average 56.3 years). We performed a biochemical examination including a microbiological urine examination. After admission, we immediately began the empirical antibacterial treatment. Results: The clinical picture was dominated by pain, prostate enlargement and failure of micturition in 185 (96.4\%) patients; 14 (7.3\%) patients had acute urinary retention where epicystostomy was necessary. Fever occurred in 177 (92.2\%) patients and Escherichia coli was the most frequent etiological agent detected in 103 (53.7\%) patients followed by Pseudomonas aeruginosa and Klebsiella species. In $16(8.3 \%)$ patients we found 2 bacteriological strains. Sterile urine was found in 65 (33.8\%) patients. Fluoroquinolones, aminoglycosides and cephalosporins were used most frequently. 147 (76.6\%) patients were treated according to the
\end{abstract}

microbiological results. Overview of resistance shows an increase in resistance to fluoroquinolones. In 14 (7.3\%) patients there was progression to urosepsis. Conclusion: Clinicians should consider local drug-resistance patterns when choosing antibiotics and should adjust the therapy on the basis of bacterial cultures and local sensitivity to antibiotics.

Copyright ๑ 2012 S. Karger AG, Basel

\section{Introduction}

The prevalence of prostatitis in humans is approximately $8.2 \%$ (range 2.2-9.7\%) [1]. The National Institutes of Health (NIH) defined four categories of prostatitis: acute bacterial, chronic bacterial, chronic prostatitis/ chronic pelvic pain syndrome, and asymptomatic. Acute bacterial prostatitis (ABP) as an acute illness requires immediate treatment and sometimes must be treated on an inpatient basis because it can lead to the complications due to urosepsis [2]. In this article, we deal with the clinical presentation, the current microbiological profile, sensitivity to antibiotics and treatment in male patients (patients) with ABP.

\section{KARGER \\ Fax +4161306 1234 \\ E-Mail karger@karger.ch}

www.karger.com
(C) 2012 S. Karger AG, Basel

0042-1138/12/0894-0445\$38.00/0

Accessible online at:

www.karger.com/uin
Assoc. Prof. Dr. med. Vincent Nagy, PhD

Department of Urology, P.J. Šafárik University

Medical Faculty and L. Pasteur University Hospital, SNP 1

SK-04011 Košice (Slovak Republic)

E-Mail vincent.nagy@upjs.sk 


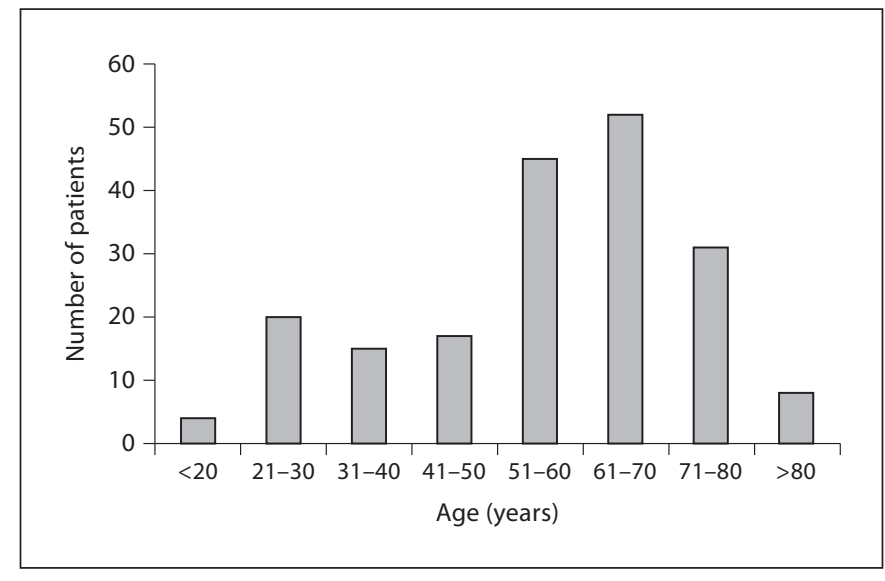

Fig. 1. Distribution of patients by age $(n=192)$.

Table 1. Results of microbiological examination of the urine $(\mathrm{n}=$ 143 bacterial strains)

\begin{tabular}{lc}
\hline & $\mathrm{n}=192$ \\
\hline Sterile urine & $65(33.8 \%)$ \\
E. coli & $103(53.6 \%)$ \\
P. aeruginosa & $10(5.2 \%)$ \\
Klebsiella species & $9(4.7 \%)$ \\
S. epidermidis & $6(3.1 \%)$ \\
P. mirabilis & $4(2.1 \%)$ \\
Enterobacter & $3(1.6 \%)$ \\
Enterococcus & $3(1.6 \%)$ \\
S. haemolyticus & $2(1.0 \%)$ \\
Acinetobacter & $2(1.0 \%)$ \\
S. beta haemolyticus & $1(0.5 \%)$ \\
\hline
\end{tabular}

\section{Patients and Methods}

In the period 1.1.2003-31.12.2010 at the Department of Urology in Košice, Slovak Republic, we treated 12,725 patients on an inpatient basis, of whom 192 (1.5\%) were diagnosed with ABP. Age range of $A B P$ patients was from 18 to 85 years (average age 56.3 years). The distribution of the patients according to age is shown in figure 1. For all the patients, we performed standard blood biochemistry, semiquantitative urine examination by the dip stick method, urinary sediment and microbiological urine examination. For microbiological examination, urine was collected as a 'clean catch-midstream' specimen. We usually use a $10-\mathrm{ml}$ sterile test tube. The urine specimens were immediately transported to the Department of Microbiology, where they use antibiotic discs. Antibiotics discs are antibiotic-impregnated paper discs used in Kirby-Bauer antibiotic testing to determine the susceptibility to specific antibiotics. Patients with a diagnosis of ABP were urgently admitted to the Department of Urology. To prevent delay in treatment, empirical intravenous antibiotic treatment is administered.

\section{Results}

The clinical picture was dominated by subjective pain in the prostate and perineum, edema of the prostate and failure of micturition in 185 (96.4\%) patients; 14 (7.3\%) patients had acute urinary retention which required epicystostomy. Fever occurred in 177 (92.2\%) patients. Fever lasted for 3.57 days on average (from 2 to 11 days). In 158 $(82.3 \%)$ patients, positive urinary sediment was confirmed microscopically. Sterile urine was found in 65 $(33.8 \%)$ patients. Of these 35 (18.2\%) patients with negative urine microbiology, antibiotics were administered before admission to our department by their general practitioners. $26(13.5 \%)$ patients had acute bacterial prostatitis after transrectal prostate biopsy, before which they had received antibiotics prophylactically. Of these, $17(8.8 \%)$ had negative urine microbiology. The other 9 (4.7\%) patients had proven Escherichia coli and Enterococcus faecalis infections. 13 (6.8\%) patients had negative microbiological findings without apparent cause. Amongst the 127 patients that had positive urine culture, 143 bacterial strains were found (table 1). Two bacteriological strains were found in $16(8.3 \%)$ patients and E. coli was the most common micro-organism identified in 103 $(\mathrm{n}=192 ; 53.7$ and $72 \%$ if $\mathrm{n}=143)$ patients, of them $12(6.3$ and $8.4 \%)$ patients had E. coli haemolyticus. Then followed Pseudomonas aeruginosa (10; 5.2 and 7\%), Klebsiella species (9; 4.7 and 6.3\%), Staphylococcus epidermidis (6; 3.1 and $4.2 \%)$, Proteus mirabilis $(4 ; 2.1$ and $2.8 \%)$, Enterobacter and Enterococcus (3; 1.6 and 2.1\%), Staphylococcus haemolyticus and Acinetobacter (2; 1 and 1.4\%) and finally Streptococcus beta haemolyticus (1; 0.5 and $0.7 \%)$. An overview of the resistance to antimicrobial drugs in ABP patients is shown in table 2. Fluoroquinolones (pefloxacin, ciprofloxacin) and aminoglycosides (gentamicin) were the most frequently used antibiotics. Individual representation of the antibiotic therapy is shown in table 3 . The clinical condition improved for 45 (23.4\%) patients after empirical antibiotic treatment. In $147(76.6 \%)$ patients, we adjusted the treatment according to the results of the microbiological examinations or we added another antimicrobial agent. In 14 (7.3\%) patients there was progression of the infection and the formation of urosepsis.

After discharge from the hospital and completion of oral antibiotic therapy, the patients underwent three microbiological examinations of the urine, the final with sterile results. After the treatment, we found no patient suffering from chronic bacterial prostatitis. However, 34 (17.7\%) patients had ABP relapse. In 2008-2010, the PSA 
Table 2. Sensitivity of bacterial strains to antibiotics in patients with ABP

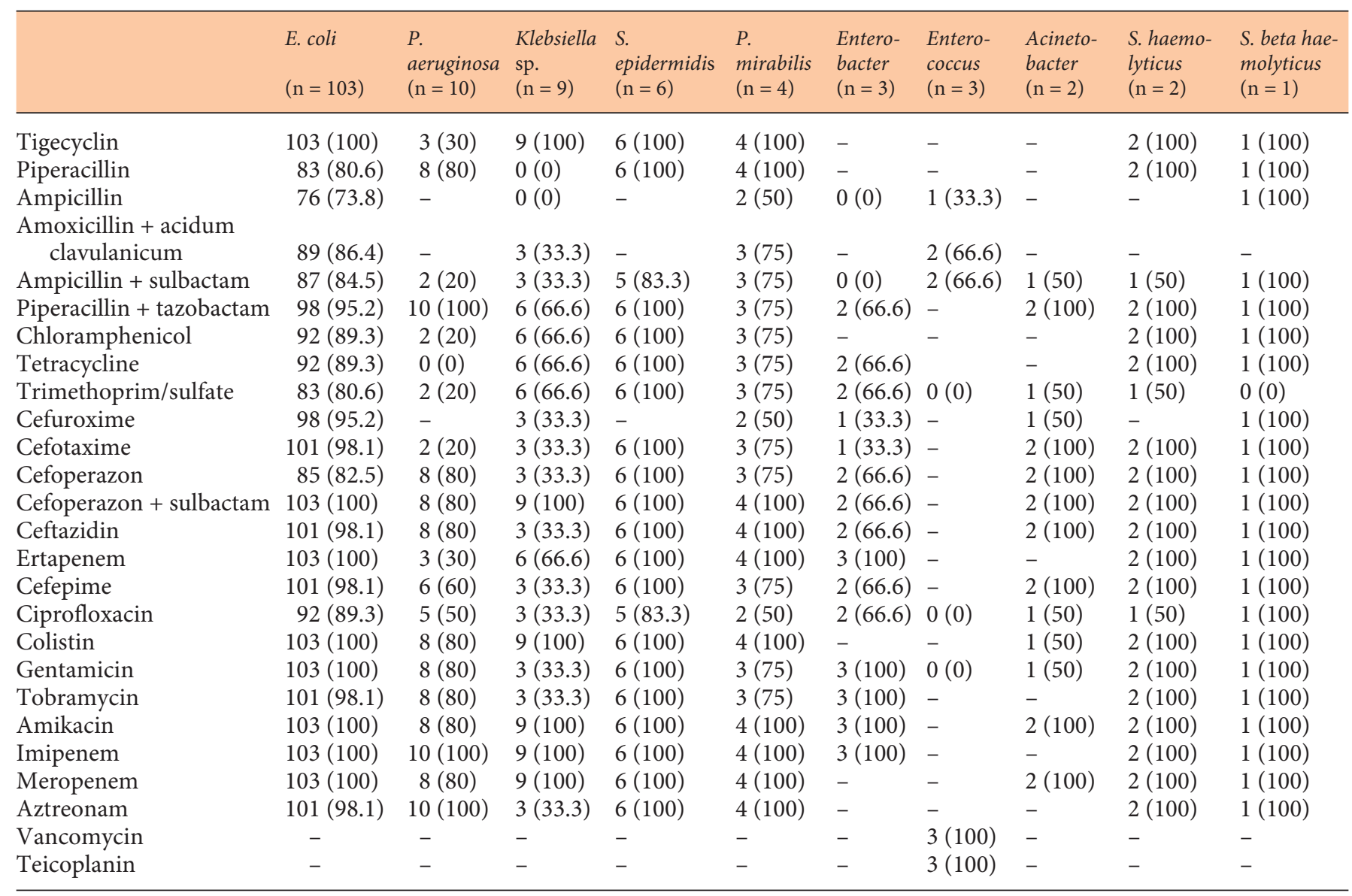

Figures in parentheses are percentages.

value was examined before and after treatment (the period of monitoring 4-38 months, average 25.7 months) in $62(32.3 \%)$ patients with ABP, aged $24-85$ years (mean age 59.8 years). The serum TPSA at the beginning of the disease was in the range of $0.59-151 \mathrm{ng} / \mathrm{ml}$ (average $21.26 \mathrm{ng} /$ $\mathrm{ml})$. At the end of the period, TPSA ranged from 0.003 to $43.18 \mathrm{ng} / \mathrm{ml}$ (mean $3.39 \mathrm{ng} / \mathrm{ml}$ ). The average decrease in TPSA was $18.08 \mathrm{ng} / \mathrm{ml}$ (range $0.12-149 \mathrm{ng} / \mathrm{ml}$ ). In 14 (23\%) patients, we took a biopsy of the prostate due to the persistence of higher TPSA values above the reference value. In 5 (35\%) of them, the biopsy confirmed prostate cancer. Four patients underwent hormonal therapy and radiation therapy, and 1 patient underwent radical retropubic prostatectomy.
Table 3. Use of antibiotics according to the representation of the active substance in our group of patients

\begin{tabular}{lc}
\hline & $\mathrm{n}=192$ \\
\hline Pefloxacin & $46(23.9 \%)$ \\
Ciprofloxacin & $45(23.4 \%)$ \\
Gentamicin & $37(19.3 \%)$ \\
Cefotaxime & $18(9.4 \%)$ \\
Cefuroxime & $12(6.4 \%)$ \\
Colimycine & $7(6.2 \%)$ \\
Ampicillin + sulbactam & $2(1.1 \%)$ \\
Piperacillin & $2(1.1 \%)$ \\
Metronidazole & $2(1.1 \%)$ \\
Cefoprerazone + sulbactam & $1(0.5 \%)$ \\
\hline
\end{tabular}




\section{Discussion}

In patients with $\mathrm{ABP}$, there are signs of lower urinary tract infection (UTI) and sometimes generalized sepsis. It is assumed that the most common route of infection of the prostate is the reflux of infected urine from the urethra into the prostate glands [2]. ABP is caused predominantly by aerobic Gram-negative pathogens (90\%), especially E. coli, which caused $50-80 \%$ of cases. Among Gram-positive bacteria (10\%), the most frequent were Enterococcus and Staphylococcus aureus. The increasing prevalence of Gram-positive pathogens may represent a changing disease epidemiology due to treatment. Some cases of prostatitis are caused by atypical pathogens $[3,4]$. Finally, sterile urine in ABP is probably attributed to the use of previous antibiotic therapy prior to the collection of urine for microbiological testing, or other unknown factors. Etienne et al. [5] investigated a series of 371 patients with ABP. Half of the patients were initially treated with a combination of antibiotics. More than $80 \%$ of the combinations included aminoglycosides; $56 \%$ of the combinations included a third-generation cephalosporin. The empirical and adapted antibiotic treatments were inadequate for 16 and $7 \%$ of the total patients, respectively. These results are in many ways similar to our conclusions. On the other hand, Lee et al. [6], from a total of 144 patients with ABP, found positive urine culture only in 51 (35.4\%) patients, most often E. coli. They concluded that in the treatment of ABP, the use of empirical antibiotics can be expected to have sufficient effects regardless of bacterial culture. A multicenter retrospective study recently revealed that community-acquired $\mathrm{ABP}$ were 3 times more common than nosocomial infections. These etiological agents were associated with higher microbiological and clinical failure rates [5]. Ciprofloxacinresistant pathogens and nosocomial acquisition or prior instrumentation were associated with increased antibiotic resistance and higher rates of clinical failure [7]. Another question is whether patients with ABP should not have blood cultures done. Etienne et al. [8] evaluated the diagnostic and prognostic values of blood cultures for 347 ABP patients. Blood cultures were positive for $21 \%$ of patients and contributed to the microbiological diagnosis for $5 \%$. Most UTIs are caused by strains of uropathogenic E. coli (UPEC) [9]. For genital infections, the pharmacokinetic properties of the antibiotics should especially be considered [10]. With the aid of a small number of additional diagnostic criteria, antibiotic treatment can be provided more specifically and thus more effectively [11]. Compared with concentrations in plasma, drug levels are generally higher in urine, similar in seminal fluid and prostatic tissue, and lower (albeit therapeutic) in prostatic fluid. In humans, alkaline drugs (e.g. trimethoprim and clindamycin) undergo ion trapping, which leads to high prostatic concentrations. Acidic drugs, such as betalactams, achieve lower levels, but more drugs are in the active unionized state. Fluoroquinolone resistance is a growing problem which generally requires treatment with a third-generation cephalosporin (e.g. ceftazidime or ceftriaxone) or a carbapenem (e.g. imipenem or ertapenem). Although penicillin $G$ achieves poor prostatic concentrations, piperacillin has good levels and has been used successfully to treat ABP. Cephalosporins can attain therapeutic levels in prostatic fluid or tissue. Aztreonam, imipenem and some aminoglycosides can attain levels in prostatic tissue that exceed the minimum inhibitory concentrations of most Enterobacteriaceae. Prostatic concentrations of minocycline and doxycycline are at least $40 \%$ of the corresponding serum concentrations. Erythromycin, and probably other macrolides, as well, can develop high prostate concentrations. Clindamycin and trimethoprim readily enter the prostatic fluid, and levels of these drugs in prostatic fluid may exceed levels in plasma. The prostatic concentration of sulfamethoxazole is much lower, raising doubts that it synergizes with trimethoprim. Nitrofurantoin prostatic levels are likely nontherapeutic. For systemically ill patients with ABP, parenteral antibiotic therapy is preferable, at least initially. The experience favors empirical treatment with a broad-spectrum beta-lactam drug - either a penicillin (e.g. piperacillin-tazobactam) or a cephalosporin (e.g. cefotaxime or ceftazidime) - perhaps combined with an aminoglycoside for patients who are severely ill or who have recently received antibiotic therapy. Clinicians should consider local drug-resistance patterns when choosing antibiotics, especially with the emergence of extended-spectrum beta-lactamase-producing strains in complicated UTIs, and should adjust the therapy on the basis of culture results. Clinically stable patients may be treated with oral therapy which is also our experience. Duration of therapy for ABP is usually 2 weeks, although it can be continued for up to 4 weeks for severe illness or treatment of patients with concomitant bacteria [3]. Careful antibiotic policies help protect against the resistance caused by antibiotic misuse.

Previous antimicrobial use and prophylaxis with fluoroquinolone are correlated with a higher prevalence of fluoroquinolone and ceftriaxone resistance and extended-spectrum beta-lactamases production. A single dose of ceftriaxone without short-course fluoroquinolone use 
is recommended as antibiotic prophylaxis in transrectal ultrasound-guided needle biopsy of the prostate [12]. Data also demonstrated different tendencies of the antibiotic susceptibilities of uropathogens according to UTI categorizations and they could be useful for planning UTI treatments [13]. One of the newer studies assessed the effects of a 4-week levofloxacin course on PSA in asymptomatic men with elevated PSA and a decision to carry out a prostate biopsy. Levofloxacin treatment resulted in an overall decrease in PSA for asymptomatic men with PSA in the 4-10 ng/dl range. PSA changes, however, were not significantly different between patients with prostate cancer and noncancer patients. Prostate cancer was detected in $20 \%$ of patients with a clinically relevant PSA decline [14]. An inflammatory pattern at primary biopsy is not associated with a decrease in prostate cancer incidence at repeated saturation prostate biopsy; therefore, only an accurate clinical evaluation including more parameters (i.e. urinary PCA3) could hopefully select men who need to undergo rebiopsy in the presence of persistent suspicion of cancer [15]. Evaluation of the preventive effect of finasteride on chronic bacterial prostatitis in Wistar rats suggest the possibility that finasteride has a preventive effect on development of chronic bacterial prostatitis, although there is as yet no consensus on the mechanism of this effect [16].

Therefore, in our study we initially used standard antibiotics. E. coli in patients with ABP has been sensitive to most of the antibiotics. Sensitivity to ciprofoxacin reached almost $90 \%$ as opposed to $55 \%$ sensitivity in the outline for the year 2011. P. aeruginosa had $100 \%$ sensitivity to piperacillin, imipenem and aztreonam; $80 \%$ sensitivity for gentamicin and third-generation cephalosporins. Ciprofloxacin resistance accounted for $50 \%$ of the strains, and was even lower (42\%) among other patients hospitalized at the Department. Klebsiella species had the best sensitivity to tigecyklin, cefoperazon + sulbactam, colistin, amikacin, imipenem and meropenem; for the other patients Proteus strains had a similar sensitivity as Klebsiella species. Sensitivity to antibiotics for other bacterial strains can be found in the individual tables. Despite the sensitivity, at the Department of Urology ABP treatment using ciprofloxacin (also in combination with other antibiotics - gentamicin, amikacin, third-generation cephalosporins) is sufficiently effective. An overview of resistance against antimicrobial drugs shows an increase in resistance to fluoroquinolones. This fact is reflected in the need to use a different product for antibacterial activity. In the selection of antibiotics, it is therefore necessary to take into account the results of resistance against antimicrobial drugs.

\section{Conclusion}

For systemically ill patients with ABP, parenteral antibiotic therapy is preferred, at least initially. Fluoroquinolones were the drugs of first choice. The experience favors empirical treatment with a broad-spectrum beta-lactam drug - either piperacillin, tazobactam or a cephalosporin (e.g. cefotaxime or ceftazidime) - perhaps combined with an aminoglycoside for patients who are severely ill or who have recently received antibiotic therapy. Clinicians should consider local drug-resistance patterns when choosing antibiotics, especially with the emergence of extended-spectrum beta-lactamase-producing strains in complicated UTIs, and should adjust the therapy on the basis of culture results. Clinically stable patients may be treated after parenteral treatment with oral therapy. Duration of therapy for $\mathrm{ABP}$ is usually 2 weeks, although it can be continued for up to 4-8 weeks for severe illness or treatment of patients with concomitant bacteriuria.

\section{Disclosure Statement}

The authors declare that they have no competing interests.

\section{References}

1 Krieger JN, Lee SW, Jeon J, Cheah PY, Liong ML, Riley DE: Epidemiology of prostatitis. Int J Antimicrob Agents 2008;31(suppl 1): 585-590.

-2 Sharp VJ, Takacs EB, Powell CR: Prostatitis: diagnosis and treatment. Am Fam Physician 2010;82:397-406.

-3 Lipsky BA, Byren I, Hoey CT: Treatment of bacterial prostatitis. Clin Infect Dis 2010;50: 1641-1652.

Acute Bacterial Prostatitis in Humans
4 Cunningham KA, Beagley KW: Male genital tract chlamydial infection: implications for pathology and infertility. Biol Reprod 2008; 79:180-189.

5 Etienne M, Chavanet P, Sibert L, Michel F, Levesque $\mathrm{H}$, Lorcerie B, Doucet J, Pfitzenmeyer P, Caron F: Acute bacterial prostatitis: heterogeneity in diagnostic criteria and management. Retrospective multicentric analysis of 371 patients diagnosed with acute prostatitis. BMC Infect Dis 2008;8:12.
6 Lee SJ, Lee DH, Park YY, Shim BS: A comparative study of clinical symptoms and treatment outcomes of acute bacterial prostatitis according to urine culture. Korean J Urol 2011;52:119-123.

7 Ha US, Kim ME, Kim CS, Shim BS, Han CH, Lee SD, Cho YH: Acute bacterial prostatitis in Korea: clinical outcome, including symptoms, management, microbiology and course of disease. Int J Antimicrob Agents 2008;31(suppl 1):96-101. 
8 Etienne M, Pestel-Caron M, Chapuzet C, Bourgeois I, Chavanet P, Caron F: Should blood cultures be performed for patients with acute prostatitis? J Clin Microbiol 2010; 48:1935-1938.

9 Blango MG, Mulvey MA: Persistence of uropathogenic Escherichia coli in the face of multiple antibiotics. Antimicrob Agents Chemother 2010;54:1855-1863.

10 Wagenlehner FM, Wullt B, Perletti G: Antimicrobials in urogenital infections. Int J Antimicrob Agents 2011;38(suppl):3-10.

-11 Schmiemann G, Kniehl E, Gebhardt K, Matejczyk MM: The diagnosis of urinary tract infection. Dtsch Arztebl Int 2010;107: 361-367.
2 Siriboon S, Tiengrim S, Taweemongkongsup T, Thamlikitkul V, Chayakulkeeree M: Prevalence of antibiotic resistance in fecal flora of patients undergoing transrectal ultrasoundguided prostate biopsy in Thailand. Urol Int 2012;88:187-193.

13 Yamamichi F, Shigemura K, Matsumoto M, Nakano Y, Tanaka K, Arakawa S, Fujisawa $\mathrm{M}$ : Relationship between urinary tract infection categorization and pathogens' antimicrobial susceptibilities. Urol Int 2012;88: 198-208.
14 Torky M, Mosharafa A, Emran A, Kamal A, Abdelhamid M: Antimicrobial therapy for asymptomatic patients with elevated prostate-specific antigen: can the change in prostate-specific antigen reliably guide prostate biopsy decisions? Urol Int 2011;87:416-419.

15 Pepe P, Aragona F: Does an inflammatory pattern at primary biopsy suggest a lower risk for prostate cancer at repeated saturation prostate biopsy? Urol Int 2011;87:171174.

16 Lee CB, Ha US, Yim SH, Lee HR, Sohn DW, Han $\mathrm{CH}$, Cho YH: Does finasteride have a preventive effect on chronic bacterial prostatitis? Pilot study using an animal model. Urol Int 2011;86:204-209. 